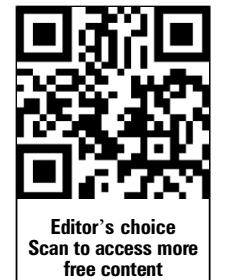

- Additional material is published online only. To view please visit the journal online (http://dx.doi.org/10.1136/ jclinpath-2012-201335).

1 Institute of Pathology, University Medical Center, Hamburg-Eppendorf, Germany ${ }^{2}$ Martini Clinic, Prostate Cancer Center, University Medical Center, Hamburg-Eppendorf, Germany

${ }^{3}$ Department of Urology, Section for Translational Prostate Cancer Research, University Medical Center, Hamburg-Eppendorf, Germany

\section{Correspondence to} Uwe Michl, Martini-Clinic, Prostate Cancer Center, University Medical Center 20246, Hamburg-Eppendorf Germany;

michl@uke.de

Received 6 November 2012 Revised 31 December 2012 Accepted 4 February 2013 Published Online First 4 April 2013

\title{
Strong expression of the neuronal transcription factor FOXP2 is linked to an increased risk of early PSA recurrence in ERG fusion-negative cancers
}

\author{
Laura Stumm, ${ }^{1}$ Lia Burkhardt, ${ }_{1}^{1}$ Stefan Steurer, ${ }_{1}^{1}$ Ronald Simon, ${ }^{1}$ Meike Adam, ${ }^{2}$ \\ Andreas Becker, ${ }^{2}$ Guido Sauter, ${ }^{1}$ Sarah Minner, ${ }^{1}$ Thorsten Schlomm, ${ }^{2,3}$ \\ Hüseyin Sirma, ${ }^{1}$ Uwe Michl ${ }^{2}$
}

ABSTRACT
Background and aims Transcription factors of the forkhead box P (FOXP1-4) family have been implicated in various human cancer types before. The relevance and role of neuronal transcription factor FOXP2 in prostate cancer is unknown.

Methods A tissue microarray containing samples from more than 11000 prostate cancers from radical prostatectomy specimens with clinical follow-up data was analysed for FOXP2 expression by immunohistochemistry. FOXP2 data were also compared with pre-existing ERG fusion (by fluorescence in situ hybridisation and immunohistochemistry) and cell proliferation (Ki67 labelling index) data.

Results There was a moderate to strong FOXP2 protein expression in basal and secretory cells of normal prostatic glands. As compared with normal cells, FOXP2 expression was lost or reduced in $25 \%$ of cancers. Strong FOXP2 expression was linked to advanced tumour stage, high Gleason score, presence of lymph node metastases and early tumour recurrence ( $p<0.0001$; each) in ERG fusion-negative, but not in ERG fusion-positive cancers. High FOXP2 expression was linked to high Ki67 labelling index $(p<0.0001)$ in all cancers irrespective of ERG fusion status.

Conclusions These data demonstrate that similar high FOXP2 protein levels as in normal prostate epithelium exert a 'paradoxical' oncogenic role in 'non fusion-type' prostate cancer. It may be speculated that interaction of FOXP2 with members of pathways that are specifically activated in 'non fusion-type' cancers may be responsible for this phenomenon.

\section{INTRODUCTION}

Transcription factors of the forkhead box $\mathrm{P}$ (FOXP1-4) family have been implicated in various human cancer types before. ${ }^{1}$ For example, FOXP1 is a tumour suppressor in breast cancer ${ }^{2}$ and has been suggested to play a role in prostate cancer also. ${ }^{3}$ FOXP3 is strongly expressed in tumour invading regulatory $\mathrm{T}$ cells and in lymphomas. ${ }^{4}$ FOXP4 expression is downregulated in kidney cancer $^{5}$ and inactivated by translocation in several breast cancer cell lines. ${ }^{6}$ In a recent study, FOXP2 has also been implicated with cancer. Campbell et $a l^{7}$ found FOXP2 overexpression as a strong discriminator between normal lymphocytes and multiple myeloma. Apart from this, FOXP2 is known to have a role in neuronal differentiation. Specifically, FOXP2 was shown as a factor influencing speech development. ${ }^{8}$ Apart from this one study in lymphoma, the role of FOXP2 expression in cancer has not been evaluated.

To determine, whether FOXP2 expression might have a role in prostate cancer, we made use of a prostate cancer tissue microarray (TMA) containing samples from more than 11000 patients with follow-up information and an attached molecular database. The data show that FOXP2 expression is often either lost/reduced or increased in prostate cancer and that FOXP2 expression is significantly linked to tumour aggressiveness, especially in nonfusion type prostate cancer.

\section{METHODS}

\section{Patients}

A TMA was made from 11152 prostatectomy specimens from patients undergoing surgery between 1992 and 2011 at the Department of Urology, and the Martini Clinics at the University Medical Center Hamburg-Eppendorf. The TMA manufacturing process was described earlier in detail. ${ }^{9}$ In short, one $0.6 \mathrm{~mm}$ core was taken from a representative tissue block from each patient. The tissues were distributed among 24 TMA blocks, each containing 129-522 cores. Clinical follow-up data were available for 9695 of the 11152 arrayed tumours. Median follow-up was 36.8 months ranging from 1 month to 219 months. None of the patients received neoadjuvant endocrine therapy. Additional (salvage) therapy was only initiated after biochemical relapse, the clinical endpoint of this study. Prostate specific antigen values were measured following surgery and recurrence was defined as a postoperative prostate specific antigen (PSA) of $0.2 \mathrm{ng} / \mathrm{ml}$ and increasing thereafter. The detailed composition of the TMA and the attached histopathological and clinical data is outlined in table 1. Presence or absence of cancer tissue was validated by immunohistochemical AMACR and 34BE12 analysis. ${ }^{10}$ More than 50 tissue spots containing normal prostate glands were also included on the TMA. The molecular database attached to this TMA contained results on ERG expression in 9628 tumours and ERG breakapart fluorescence in situ hybridisation (FISH) analysis in 6106 tumours (extended from ${ }^{11}$ ), as well as Ki67 labelling index (LI) in 7019 cancers (extended from ${ }^{10}$ ).

\section{Immunohistochemistry}

Freshly cut TMA sections were analysed in 1 day and in one experiment. The antibody anti-FOXP2 
Table 1 Composition of the prognosis tissue microarray (TMA) containing 10152 prostate cancer specimens

\begin{tabular}{|c|c|c|}
\hline & \multicolumn{2}{|c|}{ Number of patients (\%) } \\
\hline & $\begin{array}{l}\text { Study cohort } \\
\text { on TMA } \\
\text { ( } n=11152 \text { ) }\end{array}$ & $\begin{array}{l}\text { Biochemical relapse } \\
\text { among categories } \\
\text { ( } n=1824)\end{array}$ \\
\hline \multicolumn{3}{|c|}{ Follow-up (months) } \\
\hline Mean & 53.4 & - \\
\hline Median & 36.8 & - \\
\hline \multicolumn{3}{|l|}{ Age (years) } \\
\hline$<50$ & 318 & 49 \\
\hline $50-60$ & 2.768 & 460 \\
\hline $60-70$ & 6.548 & 1.081 \\
\hline$>70$ & 1.439 & 232 \\
\hline \multicolumn{3}{|c|}{ Pretreatment PSA (ng/ml) } \\
\hline$<4$ & 1.407 & 142 \\
\hline $4-10$ & 6.735 & 827 \\
\hline $10-20$ & 2.159 & 521 \\
\hline$>20$ & 720 & 309 \\
\hline \multicolumn{3}{|c|}{ Tumour stage } \\
\hline pT2 & 7.370 & 570 \\
\hline рТЗа & 2.409 & 587 \\
\hline pT3b & 1.262 & 618 \\
\hline pT4 & 63 & 49 \\
\hline \multicolumn{3}{|c|}{ Gleason grade } \\
\hline$<3+3$ & 2.859 & 193 \\
\hline $3+4$ & 1.565 & 573 \\
\hline $4+3$ & 6.183 & 849 \\
\hline$>4+4$ & 482 & 208 \\
\hline \multicolumn{3}{|c|}{ Lymph node metastasis } \\
\hline pNO & 6.117 & 1.126 \\
\hline $\mathrm{pN}+$ & 561 & 291 \\
\hline \multicolumn{3}{|c|}{ Surgical margin } \\
\hline Negative & 8.984 & 1.146 \\
\hline Positive & 1.970 & 642 \\
\hline
\end{tabular}

(rabbit polyclonal, Sigma-Aldrich, St Louis, Missouri, USA; $1 / 60$ dilution; cat\#HPA000382) was used for detection of FOXP2 expression. Slides were deparaffinised and exposed to heat-induced antigen retrieval for $5 \mathrm{~min}$ in an autoclave at $121^{\circ}$ $\mathrm{C}$ in $\mathrm{pH} 9$ buffer. Bound primary antibody was visualised using the DAKO EnVision Kit (Dako). Staining was predominantly nuclear and its intensity was estimated in a 4-step scale as negative, weak, moderate and strong staining.

\section{Statistics}

For statistical analysis, the JMP V.9.0 software (SAS Institute Inc, North Carolina, USA) was used. Contingency tables were calculated to study the association between FOXP2 expression and clinicopathological variables, and the $\chi^{2}$ (likelihood) test was used to find significant relationships. Kaplan-Meier curves were generated for PSA recurrence-free survival. The logrank test was applied to test the significance of differences between stratified survival functions. Cox proportional hazards regression analysis was performed to test the statistical independence and significance between pathological, molecular and clinical variables.

\section{RESULTS}

\section{Technical aspects}

TMA blocks with a total of 2088 tissue spots were excluded from the analysis due to insufficient immunohistochemistry (IHC) quality. FOXP2 analysis was successful in 8289 of the remaining 9064 arrayed cancers (91\%). IHC was not interpretable in 775 (9\%) tumours because lack of unequivocal tumour cells in the tissue spots or missing tissue spots on the TMA section.

\section{FOXP2 expression in normal prostate epithelium and in prostate cancer}

Intensity of FOXP2 immunostaining was moderate to strong in normal prostate epithelium and exclusively seen in the nucleus. Nuclei of basal and secretory cells were labelled, whereas no staining was seen in stromal cells. FOXP2 expression intensity was often reduced in prostate cancer as compared with normal epithelium (figure 1). FOXP2 immunostaining was found in 7779 of the 8289 analysable tumours (94\%), including 1572 (19\%) tumours with weak, 2846 (34\%) with moderate and 3361 (41\%) tumours with strong staining (table 2). Representative images are shown in figure 1. Strong FOXP2 positivity was significantly linked to advanced tumour stage, high Gleason grade and presence of lymph node metastases $(\mathrm{p}<0.0001$; each). A subset analysis in 4206 ERG fusionnegative and 3096 ERG fusion-positive cancers with FOXP2 IHC data revealed that all associations of FOXP2 with unfavourable tumour phenotype were particularly strong in ERG-negative cancers $(\mathrm{p}<0.0001$; each), but were completely lost in ERG-positive tumours. All associations of FOXP2 in all tumours and in ERG-positive and ERG-negative subgroups are summarised in table 2 and online supplementary tables S1-2.

\section{Tumour cell proliferation (Ki67LI)}

Strong FOXP2 staining was significantly linked to increased cell proliferation as measured by Ki67LI in all cancers (figure 2A; $\mathrm{p}<0.0001)$. This association was independent of the ERG fusion status (figure 2B,C; $\mathrm{p}<0.0001$; each). This association was also independent from the Gleason grade, since it held also true if tumours of identical grade were separately analysed (figure 3). Overall, these data suggest a role of FOXP2 for tumour growth in general and a direct involvement in proliferation in particular.

\section{TMPRSS2-ERG fusion status and ERG protein expression}

The relationship of FOXP2 expression and 'fusion-type' prostate cancer was analysed by two independent methods. There were subsets of 4613 cancers with available TMPRSS2-ERG fusion data obtained by FISH and there were 7302 cancers with FOXP2 and ERG IHC data. Strong FOXP2 immunostaining was more frequent in ERG IHC-positive cancers (1826/3096; 59\%) as compared with ERG IHC-negative cancers (1177/4206 (28\%), p<0.0001, figure 4A). Genomic ERG rearrangement data obtained by FISH using an ERG breakapart probe ${ }^{11}$ yielded similar results. Strong FOXP2 staining was markedly more frequent in tumours with ERG rearrangement (1315/2077 $(63 \%))$ as compared with tumours without ERG rearrangement (831/2536 (33\%), $\mathrm{p}<0.0001$, figure 4B).

\section{Prognostic relevance of FOXP2 expression}

Follow-up data were available from 7050 cancers with FOXP2 IHC data. Strong $(3+)$ FOXP2 staining was significantly linked to early biochemical recurrence in all cancers $(p<0.0001$, figure $5 \mathrm{~A}$ ). This association became even more evident in 3543 
Figure 1 Representative pictures of FOXP2 immunostaining in prostate cancer and prostate normal tissue. (A) Negative, (B) weak, (C) moderate, (D) strong staining in cancers, (D) cancer (magnified in $\mathrm{G}$ ) and adjacent normal prostate epithelium (magnified in $\mathrm{F}$ ) in the same tissue spot $(\mathrm{E})$.
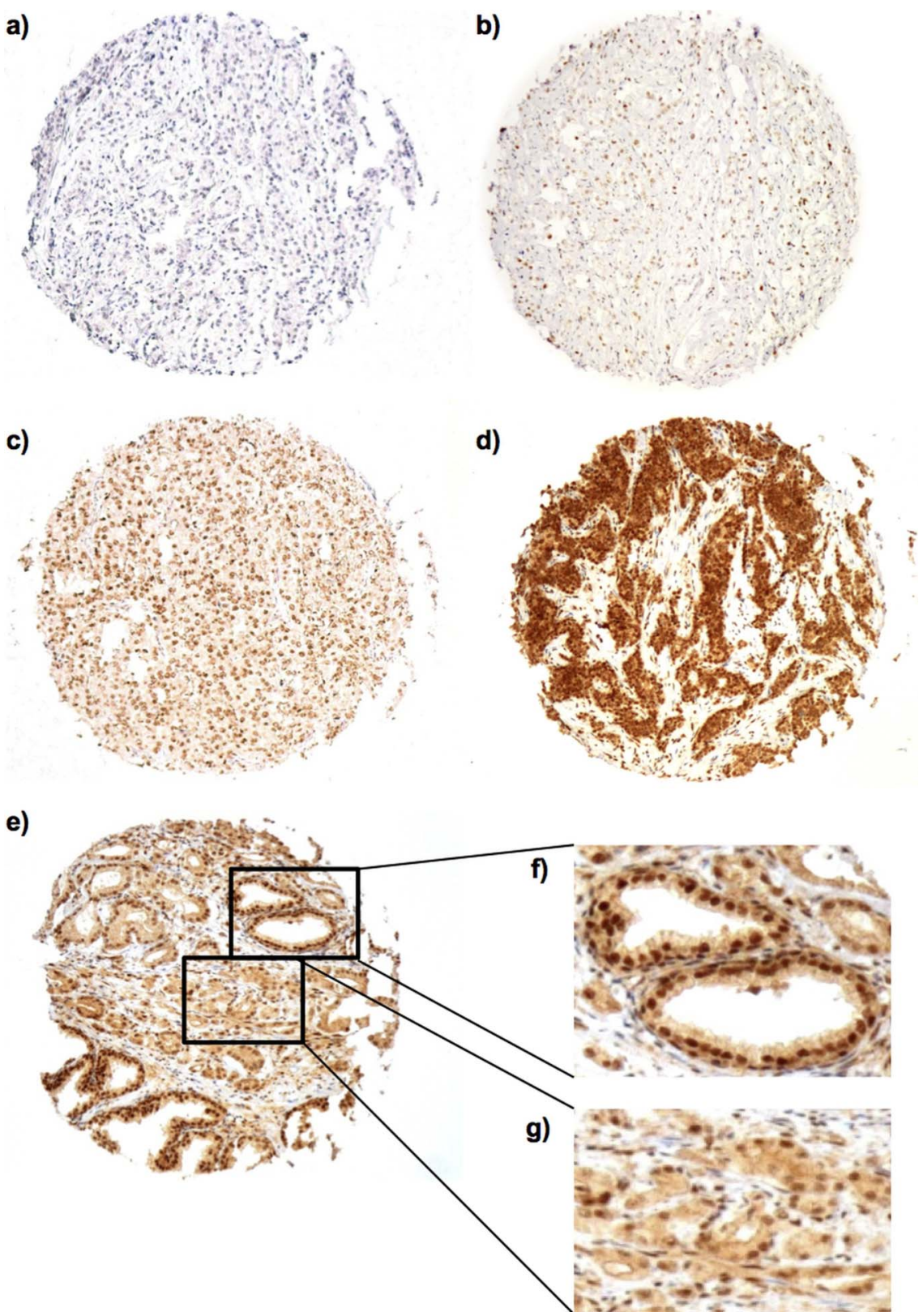

ERG-negative cancers ( $\mathrm{p}<0.0001$, figure 5B), but was completely lost in the subset of 2625 ERG fusion-positive tumours $(p=0.3887$, figure 5C). A multivariate analysis revealed that this association failed to become independent $(p=0.1033)$ from established prognostic risk factors including $\mathrm{pT}$ stage, Gleason grade and nodal stage $(\mathrm{p}<0.0001$; each) if all cancers were jointly analysed (table 3 ).

\section{DISCUSSION}

The results of our study demonstrate that high FOXP2 expression is strongly linked to adverse features of the disease in ERG fusion-negative prostate cancers. In particular, FOXP2 overexpression was linked to advanced tumour stage, high Gleason grade, and presence of lymph node metastases and early tumour recurrence.

In this study, FOXP2 was constitutively expressed at high level in the nuclei of basal and secretory cells of normal prostate glands. FOXP1-4 family members act as transcriptional repressors and regulate differentiation of neuronal and immune cells. $^{12-14}$ Thus, our findings are consistent with a general role of FOXP2 for prostate epithelial differentiation and functioning, including the proliferative basal cells and the differentiated, quiescent secretory cells. The absence of FOXP2 protein in stromal cells of the prostate fits well with results of a previous study reporting relatively low FOXP2 mRNA levels in the prostate. The stromal component can be substantial in prostate tissue and may have led to a massive dilution of mRNAs derived from prostate epithelium. Other studies reported FOXP2 mRNA expression from most other normal tissues also, with highest levels in ovary, colon, brain, liver and intestine, ${ }^{7}$ as well as developing tissues from lung, heart and gut. ${ }^{15}$ Several studies have linked FOXP2 expression to fetal and adult neuronal tissues, ${ }^{14}$ where it has been implicated in neuronal differentiation and speech development. ${ }^{8}$

The strong association between lost or reduced FOXP2 expression and favourable tumour phenotype and good prognosis was counterintuitive. It is much more common that the loss of molecular features of the normal prostate epithelium parallels tumour cell dedifferentiation and is rather associated with poor outcome. This for example applies for ALCAM, PTEN, 
Table 2 Association between the FOXP2 immunostaining (IHC) result and prostate cancer phenotype

\begin{tabular}{|c|c|c|c|c|c|c|}
\hline \multirow[b]{2}{*}{ Parameter } & \multirow[b]{2}{*}{$\begin{array}{l}\mathrm{n} \\
\text { Evaluable }\end{array}$} & \multicolumn{4}{|c|}{ FOXP2 IHC result } & \multirow[b]{2}{*}{$\mathrm{p}$ Value } \\
\hline & & $\begin{array}{l}\text { Negative } \\
(\%)\end{array}$ & $\begin{array}{l}\text { Weak } \\
(\%)\end{array}$ & $\begin{array}{l}\text { Moderate } \\
(\%)\end{array}$ & $\begin{array}{l}\text { Strong } \\
(\%)\end{array}$ & \\
\hline All cancers & 8.289 & 6 & 19 & 34 & 41 & \\
\hline \multicolumn{7}{|c|}{ Tumour stage } \\
\hline pT2 & 5.546 & 7 & 21 & 34 & 38 & $<0.0001$ \\
\hline рТЗа & 1.796 & 5 & 16 & 33 & 46 & \\
\hline pT3b & 904 & 3 & 14 & 40 & 43 & \\
\hline pT4 & 22 & 0 & 5 & 45 & 50 & \\
\hline \multicolumn{7}{|c|}{ Gleason grade } \\
\hline$<3+3$ & 1.817 & 8 & 23 & 35 & 34 & $<0.0001$ \\
\hline $3+4$ & 4.827 & 6 & 19 & 33 & 42 & \\
\hline $4+3$ & 1.220 & 4 & 16 & 36 & 45 & \\
\hline$>4+4$ & 394 & 3 & 13 & 40 & 44 & \\
\hline \multicolumn{7}{|c|}{ Lymph node metastasis } \\
\hline NO & 4.363 & 6 & 18 & 34 & 43 & $<0.0001$ \\
\hline $\mathrm{N}+$ & 429 & 4 & 10 & 40 & 46 & \\
\hline \multicolumn{7}{|c|}{ Surgical margin } \\
\hline Negative & 6.652 & 6 & 19 & 34 & 41 & 0.2967 \\
\hline Positive & 1.479 & 6 & 17 & 38 & 46 & \\
\hline
\end{tabular}

E-cadherin or CDKN2A (p16) in prostate cancer. ${ }^{16-19}$ One possible explanation for the unusual scenario, that a normal cell protein all of a sudden becomes oncogenic is that a switch of the cell metabolism has occurred in which the 'normal cell protein' obtains a completely different role or function. That FOXP2 has the potential to interfere with pathways that are related to cancer is well known. For example, FOXP2 regulates the expression of a variety of genes involved in tumour-relevant pathways such as WNT, NF-K B and IGF-1. ${ }^{20}{ }^{21}$ Moreover, FOXP2 interacts with, and regulates the activity of C-terminal binding protein 1 (CTBP1). ${ }^{22}$ Overexpression of CTBP1 promotes cell cycle progression and inhibits apoptosis by repressing the expression of known tumour suppressor and apoptosis genes such as PTEN, p16 and BAX. ${ }^{23}$ Moreover, that FOXP2 was found overexpressed in lymphoma cells as compared with their normal counterparts is further suggesting that FOXP2 can exert an oncogenic function in vivo. ${ }^{7}$

Our subset analyses in ERG-positive and ERG-negative prostate cancers appear to potentially explain the paradoxical associations of retained high FOXP2 levels with unfavourable tumour features. The most common chromosomal rearrangement known in prostate cancer fuses the androgen responsive TMPRSS2 gene to the ETS family transcription factor ERG gene. ${ }^{24}$ This alteration occurs in about $50 \%$ of prostate cancers and causes high level ERG protein expression and a variety of molecular downstream changes in affected cancers. ${ }^{24-27}$ The TMPRSS2:ERG fusion is the prototype of a number of similar rearrangements resulting in oncogenic activation of ETS genes $^{28-31}$ in prostate cancer, which have led to a molecular subclassification of fusion-negative and fusion-positive prostate cancers. Our data revealed a significant difference in frequency and prognostic impact of FOXP2 expression in fusion-type and non fusion-type prostate cancer. We found that a retained, moderate to strong FOXP2 expression was significantly linked to ERG fusion-positive cancers. Importantly, this association held true for immunohistochemical analysis of ERG expression and for FISH-based detection of ERG breakage, excluding a

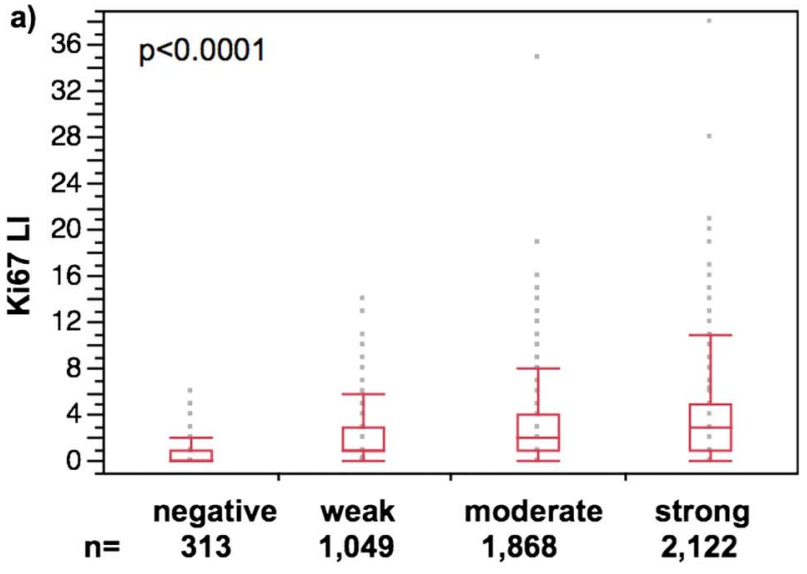

b)

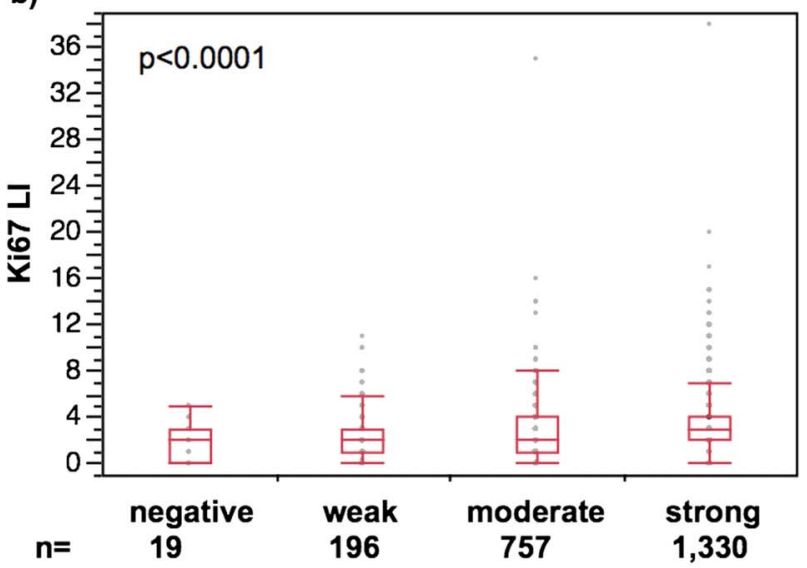

c)

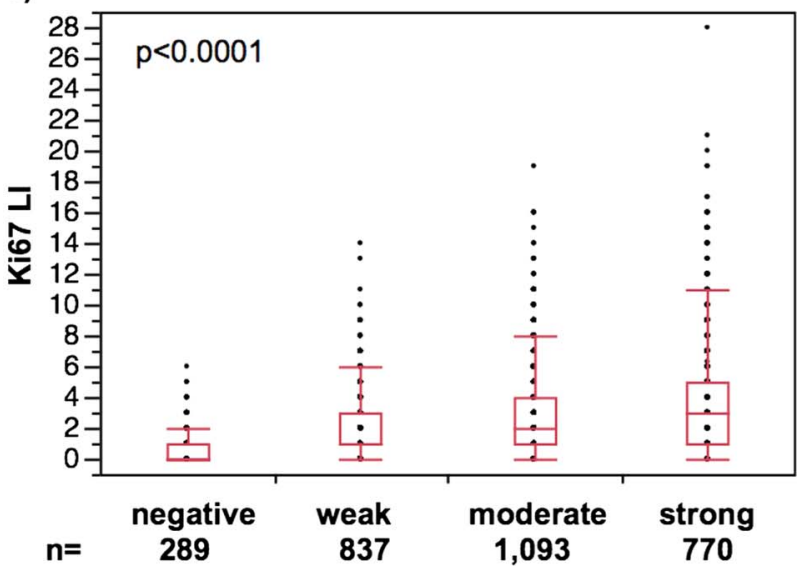

Figure 2 Association between FOXP2 immunostaining intensity and cell proliferation as measured by the Ki67 labelling index (Ki67 LI) in (A) all cancers $(n=5352),(B)$ ERG positive cancers $(n=2302)$, and (C) ERG negative cancers $(n=2989)$.

potential IHC-related artificial relationship. Strikingly, almost all adverse features connected to FOXP2 overexpression were limited to the subset of ERG-negative cancers.

This finding argues for biologically distinct consequences of FOXP2 in ERG fusion-negative and ERG fusion-positive cancers. A different role of FOXP2 in 'fusion-type' and 'non fusion-type' prostate cancer is conceivable. Alternatively, the tumour promoting effect of FOXP2 may be counteracted by ERG. ERG is normally not expressed in the prostate epithelium. Activation of ERG expression by TMPRSS2:ERG gene fusion 


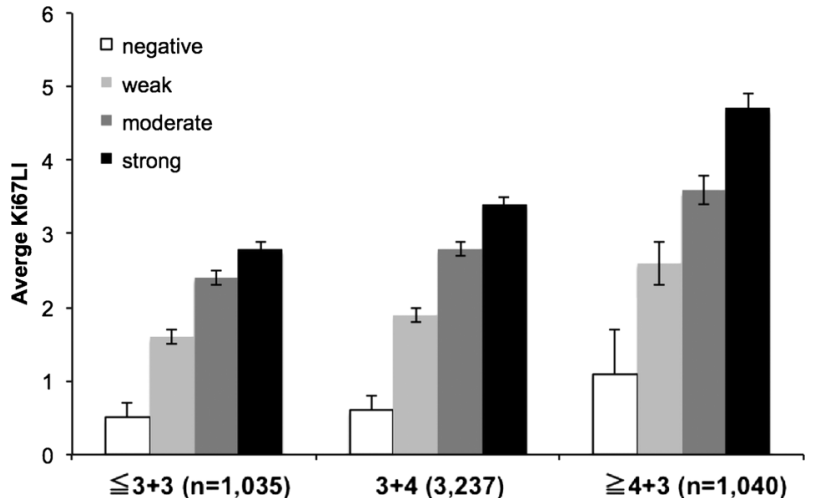

Figure 3 Significant associations between FOXP2 immunostaining and Ki67 labelling index (Ki67LI) in tumour subsets of identical Gleason grade.

results in aberrant activation of different signalling cascades in ERG fusion-positive as compared with ERG fusion-negative prostate cancers. ${ }^{27}{ }^{32-34}$ One of the best known consequences of ERG expression is activation of WNT signalling 262735 It is tempting to speculate that FOXP2 and ERG, or their target genes, interfere with pathways governing tumour aggressiveness, possibly including the WNT pathway, which is subsequently reflected by the different clinical course in ERG-positive and ERG-negative cancers. The genes or mechanisms mediating this effect remain to be identified.

Indirect evidence for substantial differences on the cell biological effect of FOXP2 depending of the gene sets activated in cancer cells is derived from the significant link of high FOXP2 expression and multiple myeloma pathogenesis. ${ }^{7}$

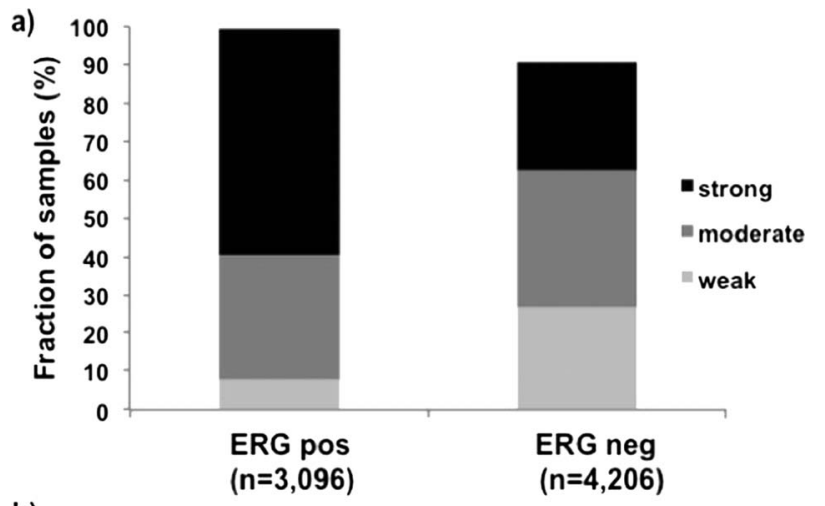

b)

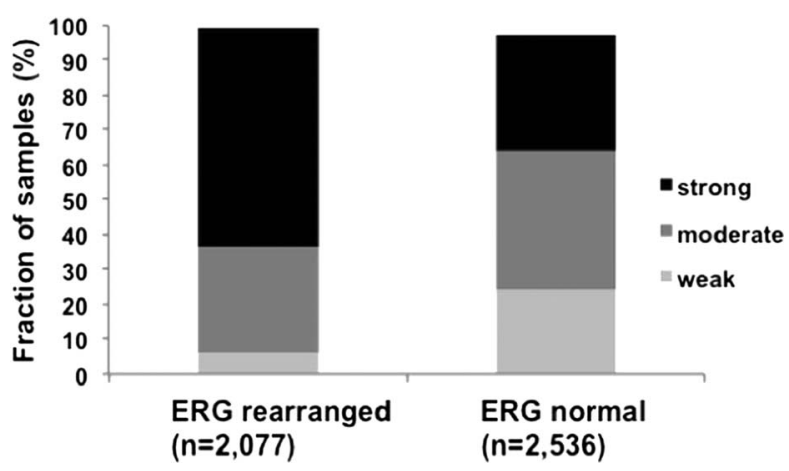

Figure 4 Association between positive FOXP2 immunostaining and ERG fusion status probed by (A) IHC and (B) FISH analysis. $p<0.0001$; each.
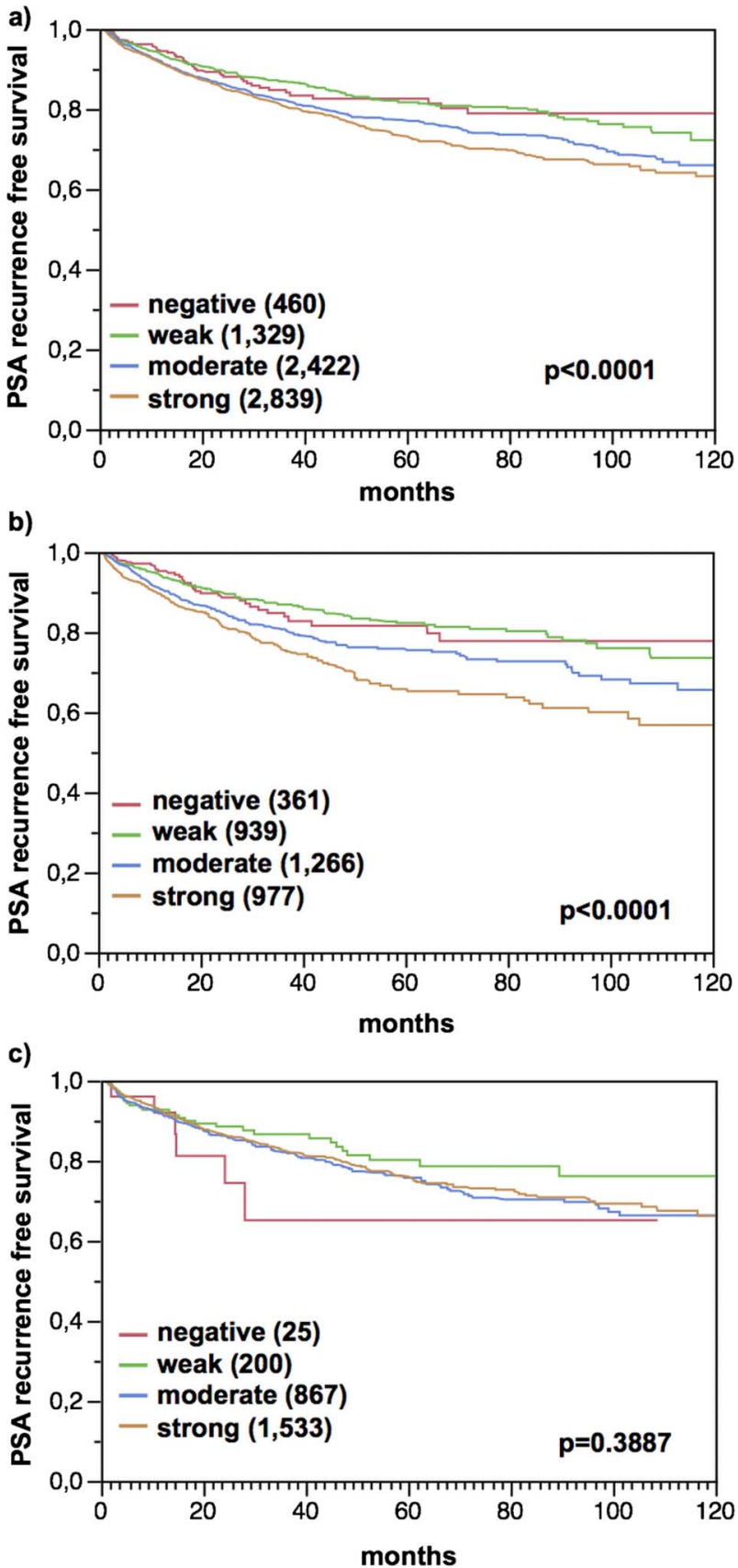

Figure 5 Association between FOXP2 immunostaining intensity and biochemical recurrence in (A) all cancers $(n=7050),(B)$ ERG-negative cancers $(n=3543)$ and (C) ERG-positive cancers $(n=2625)$.

In summary our study suggests an important role of FOXP2 for prostate cancer development and progression, and suggests biologically different effects in ERG fusion-positive and ERG fusion-negative cancers. In non fusion-type prostate cancer, high FOXP2 expression stratifies a subset of prostate cancers with a particularly poor prognosis.

Acknowledgements We thank Christina Koop, Julia Schumann, Sünje Seekamp and Inge Brandt for excellent technical assistance.

Contributors $\mathrm{LS}$ and LB conceived the study, carried out experiments and analysed data. SS, GS, SM performed the pathological review. GS, RS, HS, SM, TS and UM participated in the design of the study, statistical analysis of the data and drafting of the manuscript. All authors were involved in interpreting data, writing the paper and approving the final version of the manuscript. UM is responsible for the overall content as guarantor. 
Table 3 COX regression multivariate analysis for the prediction of biochemical recurrence

\begin{tabular}{lllll}
\hline Parameter & Factor & HR & 95\% Cl & p Value \\
\hline \multirow{2}{*}{ pT Stage } & pT3a vs pT2 & 2.3 & 1.9 to 2.8 & $<0.0001$ \\
& pT3b vs pT3a & 1.8 & 1.5 to 2.1 & \\
Gleason & pT4 vs pT3b & 1.1 & 0.5 to 2.0 & \\
& 3+4 vs $<3+3$ & 1.7 & 1.2 to 2.5 & $<0.0001$ \\
pN stage & 4+3 vs $3+4$ & 2.2 & 1.8 to 2.5 & \\
FOXP2 & N+4 vs 4+3 & 1.0 & 0.8 to 1.2 & \\
& N+ vs N0 & 1.7 & 1.4 to 2.1 & $<0.0001$ \\
& Weak vs negative & 0.8 & 0.6 to 1.2 & 0.1033 \\
& Moderate vs weak & 1.2 & 0.9 to 1.4 & \\
\hline
\end{tabular}

\section{Take-home message}

- The forkhead box transcription factor FOXP2 is abundantly expressed in a broad variety of human tissues.

Physiologically, FOXP2 regulates transcriptional programs required for neuronal differentiation, but several studies have also suggested a role in cancer biology.

- Employing immunohistochemistry, we found largely comparable levels of FOXP2 expression in normal and cancerous prostate. Paradoxically, FOXP2 expression was linked to agressive tumor features, including advanced stage, high Gleason grade, increased proliferation and early biochemical recurrence.

- The adverse effect of FOXP2 expression was limited to prostate cancers lacking TMPRSS2:ERG fusion, suggesting that these cancers may activate pathways that interact with FOXP2 in promoting tumor progression.

Funding This work was supported by the German Research Foundation (DFG SI 572/4-1) and Federal Ministry of Education and Research (BMBF) (FKZ 01GS08189).

Competing interests None.

Provenance and peer review Not commissioned; externally peer reviewed.

\section{REFERENCES}

1 Katoh M. Human FOX gene family (Review). Int I Oncol 2004;25:1495-500.

2 Fox SB, Brown P, Han C, et al. Expression of the forkhead transcription factor FOXP1 is associated with estrogen receptor alpha and improved survival in primary human breast carcinomas. Clin Cancer Res 2004;10:3521-7.

3 Taylor BS, Schultz N, Hieronymus $\mathrm{H}$, et al. Integrative genomic profiling of human prostate cancer. Cancer Cell 2010;18:11-22.

4 Bignone PA, Banham AH. FOXP3+ regulatory T cells as biomarkers in human malignancies. Expert Opin Biol Ther 2008;8:1897-920.

5 Teufel A, Wong EA, Mukhopadhyay M, et al. FoxP4, a novel forkhead transcription factor. Biochim Biophys Acta 2003;1627:147-52.

6 Howarth $\mathrm{KD}$, Blood KA, Ng BL, et al. Array painting reveals a high frequency of balanced translocations in breast cancer cell lines that break in cancer-relevant genes. Oncogene 2008;27:3345-59.

7 Campbell AJ, Lyne L, Brown PJ, et al. Aberrant expression of the neuronal transcription factor FOXP2 in neoplastic plasma cells. Br I Haematol 2010;149:221-30.

8 Kang C, Drayna D. Genetics of speech and language disorders. Annu Rev Genomics Hum Genet 2011;12:145-64.
9 Minner S, Wittmer C, Graefen M, et al. High level PSMA expression is associated with early psa recurrence in surgically treated prostate cancer. Prostate 2010;71:281-8

10 Minner S, Jessen B, Stiedenroth L, et al. Low level HER2 overexpression is associated with rapid tumor cell proliferation and poor prognosis in prostate cancer. Clin Cancer Res 2010;16:1553-60.

11 Minner S, Enodien $\mathrm{M}$, Sirma $\mathrm{H}$, et al. ERG status is unrelated to PSA recurrence in radically operated prostate cancer in the absence of antihormonal therapy. Clin Cancer Res 2011;17:5878-88.

12 Mercer $F$, Unutmaz $D$. The biology of FoxP3: a key player in immune suppression during infections, autoimmune diseases and cancer. Adv Exp Med Biol 2009:665:47-59.

13 Lalmansingh AS, Karmakar $S$, Jin $Y$, et al. Multiple modes of chromatin remodeling by Forkhead box proteins. Biochim Biophys Acta 2012;1819(7):707-15.

14 Takahashi H, Takahashi K, Liu FC. FOXP genes, neural development, speech and language disorders. Adv Exp Med Biol 2009;665:117-29.

15 Schroeder DI, Myers RM. Multiple transcription start sites for FOXP2 with varying cellular specificities. Gene 2008;413:42-8.

16 Minner S, Kraetzig F, Tachezy M, et al. Low activated leukocyte cell adhesion molecule expression is associated with advanced tumor stage and early prostate-specific antigen relapse in prostate cancer. Hum Pathol 2011;42:1946-52.

17 Lotan T, Gurel B, Sutcliffe S, et al. PTEN protein loss by immunostaining: analytic validation and prognostic indicator for a high risk surgical cohort of prostate cancer patients. Clin Cancer Res 2011;17:6563-73.

18 Gravdal K, Halvorsen OJ, Haukaas SA, et al. A switch from E-cadherin to $\mathrm{N}$-cadherin expression indicates epithelial to mesenchymal transition and is of strong and independent importance for the progress of prostate cancer. Clin Cancer Res 2007;13:7003-11.

19 Chakravarti A, DeSilvio M, Zhang M, et al. Prognostic value of p16 in locally advanced prostate cancer: a study based on Radiation Therapy Oncology Group Protocol 9202. J Clin Oncol 2007;25:3082-9.

20 Yasui $\mathrm{H}$, Hideshima T, Richardson PG, et al. Novel therapeutic strategies targeting growth factor signalling cascades in multiple myeloma. Br J Haematol 2006:132:385-97.

21 Podar K, Chauhan D, Anderson KC. Bone marrow microenvironment and the identification of new targets for myeloma therapy. Leukemia 2009;23:10-24.

22 Li S, Weidenfeld J, Morrisey EE. Transcriptional and DNA binding activity of the Foxp $1 / 2 / 4$ family is modulated by heterotypic and homotypic protein interactions. Mol Cell Biol 2004;24:809-22.

23 Chinnadurai $\mathrm{G}$. The transcriptional corepressor CtBP: a foe of multiple tumor suppressors. Cancer Res 2009;69:731-4.

24 Tomlins SA, Rhodes DR, Perner $S$, et al. Recurrent fusion of TMPRSS2 and ETS transcription factor genes in prostate cancer. Science 2005;310:644-8.

25 Tomlins SA, Laxman B, Varambally $S$, et al. Role of the TMPRSS2-ERG gene fusion in prostate cancer. Neoplasia 2008;10:177-88.

26 Gupta S, Iljin K, Sara H, et al. FZD4 as a mediator of ERG oncogene-induced WNT signaling and epithelial-to-mesenchymal transition in human prostate cancer cells. Cancer Res 2010;70:6735-45.

27 Brase JC, Johannes M, Mannsperger $\mathrm{H}$, et al. TMPRSS2-ERG -specific transcriptional modulation is associated with prostate cancer biomarkers and TGF-beta signaling. BMC Cancer 2011;11:507.

28 Hermans KG, Boormans JL, Gasi D, et al. Overexpression of prostate-specific TMPRSS2(exon 0)-ERG fusion transcripts corresponds with favorable prognosis of prostate cancer. Clin Cancer Res 2009;15:6398-403.

29 Hermans KG, van der Korput HA, van Marion R, et al. Truncated ETV1, fused to novel tissue-specific genes, and full-length ETV1 in prostate cancer. Cancer Res 2008;68:7541-9.

30 Han B, Mehra R, Dhanasekaran SM, et al. A fluorescence in situ hybridization screen for E26 transformation-specific aberrations: identification of DDX5-ETV4 fusion protein in prostate cancer. Cancer Res 2008;68:7629-37.

31 Maher CA, Kumar-Sinha C, Cao X, et al. Transcriptome sequencing to detect gene fusions in cancer. Nature 2009;458:97-101.

32 Markert EK, Mizuno H, Vazquez A, et al. Molecular classification of prostate cancer using curated expression signatures. Proc Natl Acad Sci U S A 2011;108:21276-81.

33 Chen Y, Sawyers CL. Coordinate transcriptional regulation by ERG and androgen receptor in fusion-positive prostate cancers. Cancer Cell 2010;17:415-16.

$34 \mathrm{Yu}$ J, Mani RS, Cao Q, et al. An integrated network of androgen receptor, polycomb, and TMPRSS2-ERG gene fusions in prostate cancer progression. Cancer Cell 2010;17:443-54.

35 Mochmann LH, Bock J, Ortiz-Tanchez J, et al. Genome-wide screen reveals WNT11, a non-canonical WNT gene, as a direct target of ETS transcription factor ERG. Oncogene 2011;30:2044-56. 